\title{
Dephosphorylation-Induced Ubiquitination and Degradation of FMRP in Dendrites: A Role in Immediate Early mGluR-Stimulated Translation
}

\author{
Vijayalaxmi C. Nalavadi, ${ }^{1}$ Ravi S. Muddashetty, ${ }^{1}$ Christina Gross, ${ }^{1}$ and Gary J. Bassell ${ }^{1,2}$ \\ Departments of ${ }^{1}$ Cell Biology and ${ }^{2}$ Neurology, Emory University School of Medicine, Atlanta, Georgia 30322
}

Fragile $\mathrm{X}$ syndrome is caused by the loss of fragile $\mathrm{X}$ mental retardation protein (FMRP), which represses and reversibly regulates the translation of a subset of mRNAs in dendrites. Protein synthesis can be rapidly stimulated by mGluR-induced and protein phosphatase 2a (PP2A)-mediated dephosphorylation of FMRP, which is coupled to the dissociation of FMRP and target mRNAs from miRNA-induced silencing complexes. Here, we report the rapid ubiquitination and ubiquitin proteasome system (UPS)-mediated degradation of FMRP in dendrites upon DHPG (3,5-dihydroxyphenylglycine) stimulation in cultured rat neurons. Using inhibitors to PP2A and FMRP phosphomutants, degradation of FMRP was observed to depend on its prior dephosphorylation. Translational induction of an FMRP target, postsynaptic density-95 mRNA, required both PP2A and UPS. Thus, control of FMRP levels at the synapse by dephosphorylation-induced and UPS-mediated degradation provides a mode to regulate protein synthesis.

\section{Introduction}

Protein homeostasis is of universal importance in processes ranging from cell cycle to immune response to synaptic plasticity. Targeted degradation of crucial proteins by the ubiquitin proteasome system (UPS) can provide a basis for spatiotemporal regulation of various functions (Rechsteiner, 1987; Hershko and Ciechanover, 1998). UPS action can be rapid and is regarded as a type of signaling event (Pierce et al., 2009; Rinetti and Schweizer, 2010). The role of UPS is particularly important in postmitotic neurons having to continuously modify protein composition at synapses, as evidenced by the number of "proteinopathies" in the nervous system caused by susceptibility of neurons to UPS abnormalities (Ross and Poirier, 2004). Angelman syndrome results from mutations in a ubiquitin ligase whose ubiquitination of Arc is necessary for AMPA receptor endocytosis (Greer et al., 2010). Inhibition of UPS leads to defects in LTP (Hegde et al., 1993; Fonseca et al., 2006; Karpova et al., 2006) as well as LTD (Colledge et al., 2003; Hou et al., 2006). The types of synaptic proteins regulated by ubiquitination include receptors and scaf-

Received 0ct. 5, 2011; revised Dec. 27, 2011; accepted Jan. 1, 2012

Author contributions: V.C.N. and G.J.B. designed research; V.C.N., R.S.M., and C.G. performed research; V.C.N., R.S.M., C.G., and G.J.B. analyzed data; V.C.N. and G.J.B. wrote the paper.

This research was supported by National Institutes of Health Grant MH086405 (G.J.B), Fragile X Center Grant 3P30HD024064 (G.J.B.), and a FRAXA postdoctoral fellowship (V.C.N). We thank Lei Xing for making the PAGFPFMRP construct, Xiaodi Yao for the FLAG-mCherry-FMRP construct, and Wilfred Rossoll for the HA- ubiquitin construct. We thank Oscar Laur and the Emory Custom Cloning Core facility. We thank Alexa Mattheyses for technical support in the microscopy core (Emory Neuroscience NINDS Core Facilities P30NS055077). We thankStephen Warren for helpful discussions. We thank Stephanie Ceman for the phospho-FMRP antibody. We appreciate technical assistance by Laura Griffin, Nathan Sivanasundarum, and Christopher Newhouse.

Correspondence should be addressed to Gary J. Bassell, Emory University, Department of Cell Biology, 615 Michael Street, Atlanta, GA 30322. E-mail: gbassel@emory.edu.

DOI:10.1523/JNEUROSCI.5057-11.2012

Copyright $\odot 2012$ the authors $\quad 0270-6474 / 12 / 322582-06 \$ 15.00 / 0$ folding proteins (Colledge et al., 2003; Ehlers, 2003; Bingol and Schuman, 2004).

The regulated degradation of synaptic proteins by UPS is coordinated with activity-mediated synthesis of synaptic proteins (Steward and Schuman, 2003). Local protein synthesis provides a means to affect synaptic protein content and regulate plasticity (Wang et al., 2010). Proteins that regulate synaptic protein synthesis may also be degraded by UPS, providing additional control to regulate protein abundance at synapses. For example, NMDAR-induced degradation of MOV10, an argonaute protein, leads to disinhibition of translation by microRNAs (Banerjee et al., 2009).

Fragile X syndrome, the most common form of inherited intellectual disability, is caused by loss of fragile $\mathrm{X}$ mental retardation protein (FMRP), which often is a negative regulator of target mRNA translation important for synaptic function (Zalfa et al., 2003; Muddashetty et al., 2007; Darnell et al., 2011). Activation of gp1 metabotropic glutamate receptors (mGluRs) normally leads to a rapid and protein synthesis-dependent LTD, which is exaggerated, and protein synthesis-independent in Fmr1 KO, likely due to excess and dysregulated translation (Waung and Huber, 2009). Surprisingly, mGluR activation leads to both FMRP loss at synapses (Antar et al., 2004) and FMRP synthesis at synapses (Weiler et al., 1997). FMRP synthesis and UPS-mediated degradation of FMRP appear to be coordinately regulated in hippocampal slices (Hou et al., 2006), but the mechanism and function of activity-induced UPS-mediated degradation of FMRP are unclear. mGluR-induced protein synthesis-dependent LTD and mGluR-induced epileptogenesis required the UPS in WT neurons, which was altered in Fmr1 KO (Hou et al., 2006; Zhao et al., 2011). Since mGluR-induced translation is initiated by rapid and transient dephosphorylation of FMRP by protein phosphatase 2a (PP2A) (Narayanan et al., 2007), we sought to 

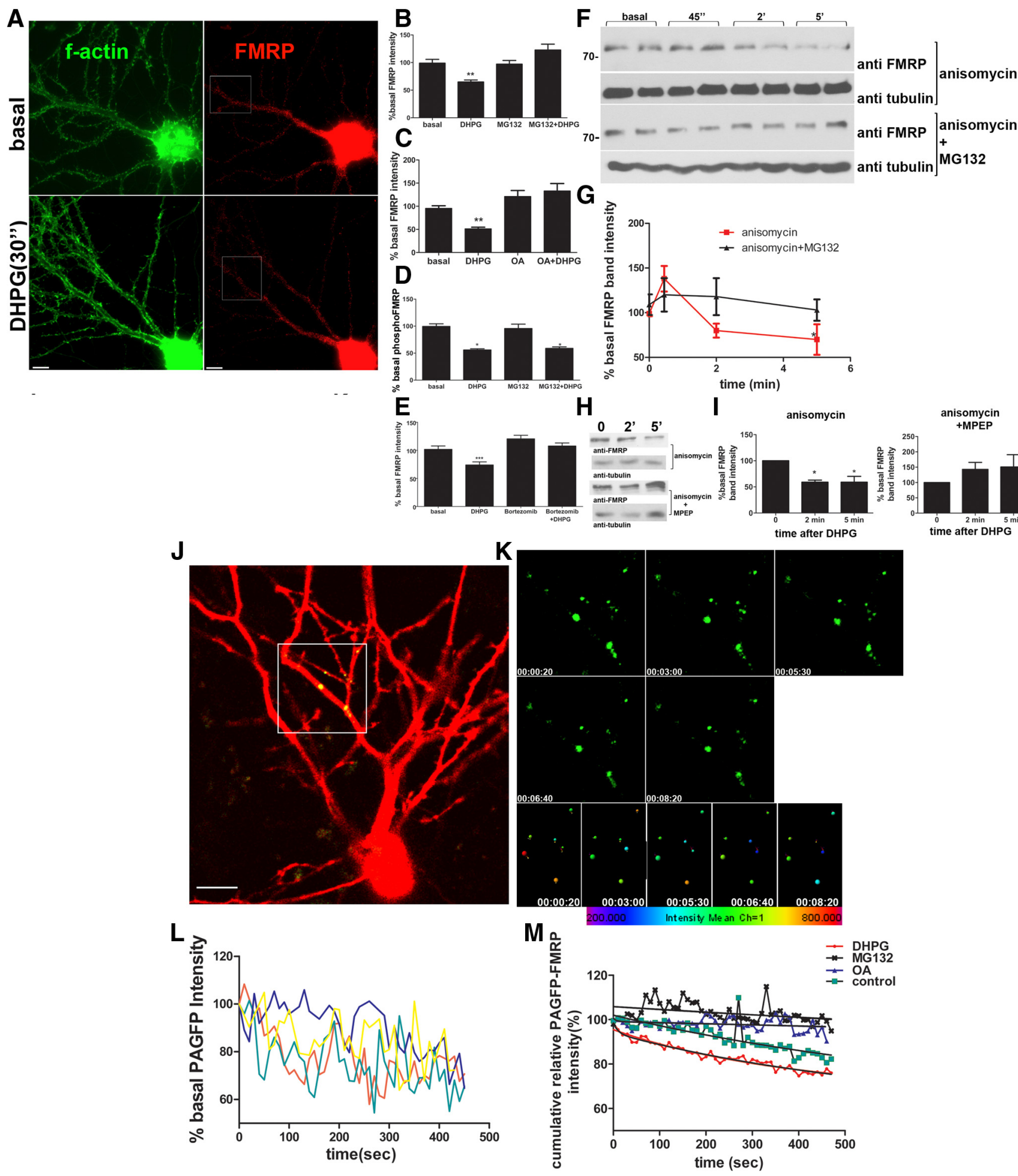

Figure 1. Rapid degradation of FMRP in dendrites and synapses by UPS in response to mGluR activation requires PP2A activity. $A$, Representative images of FMRP detected by immunofluorescence (red) in hippocampal neurons with and without DHPG treatment. Boxes indicate regions of interest for quantitation of dendritic FMRP fluorescence. Phalloidin staining (green) for $F$-actin labels neuronal morphology. $\boldsymbol{B}, \boldsymbol{C}, \boldsymbol{E}$, Quantitation of endogenous FMRP intensity from neurons treated with DHPG for $30 \mathrm{~s}$ with or without pretreatment with MG132 (B), okadaic acid $(0 \mathrm{~A})(\boldsymbol{C})$, or bortezomib $(\boldsymbol{E})$. Histograms represent normalized mean intensities for dendritic FMRP of multiple neurons per experiment. $(n=3, p<0.0001$, one-way ANOVA with Bonferroni's test, mean \pm SEM). D, Mean \pm SEM of relative intensity of phospho-FMRP signal in distal dendrites after DHPG treatment with or without MG132 $(n=3, p=0.01,0$ ne-way ANOVA). $\boldsymbol{F}$, Cortical SNSs were stimulated with DHPG for given time intervals in the presence of the protein synthesis inhibitor anisomycin, with or without MG132, and FMRP was quantified by Western blotting with an anti-FMRP antibody. G, Mean \pm SEM of normalized FMRP band intensities in anisomycin-treated SNSs as a function of time without $(n=4, p<0.001$, one-way ANOVA with Bonferroni's test) or with ( $n=$ $4, p=0.89$ ) pretreatment of the SNSs with MG132. $\boldsymbol{H}$, Synaptoneurosomes were pretreated with anisomycin only or anisomycin and MPEP before stimulation with DHPG for indicated times, and FMRP was quantified by western blot. I, Mean \pm SEM of FMRP levels in synaptoneurosomes treated with DHPG, and with or without MPEP $(n=3, p<0.05)$. J-M, mGluR stimulation of hippocampal neurons with DHPG induced a rapid UPS- and PP2A-dependent loss of locally photoactivated dendritic PAGFP-FMRP signal. J, Neurons were cotransfected with PAGFP-FMRP and mCherry (to reveal morphology), and were locally photoactivated to reveal the GFP fluorescence only in the distal dendrites before activation (Figure legend continues.) 
investigate a link with UPS-mediated degradation of FMRP. Here we show that mGluR-induced dephosphorylation of FMRP facilitates its ubiquitination and UPS-mediated degradation, which may provide a switch for rapid translation induction by mGluRs.

\section{Materials and Methods}

Cell culture. Neurons were cultured from E18 Sprague Dawley rat embryos of either sex, as described previously (Antar et al., 2004).

DNA constructs and transfections. FLAG-GFP-FMRP constructs mutated at serine 499 and F-luciferase-postsynaptic density-95 (PSD-95) UTR have been described (Narayanan et al., 2007). FLAG3XmCherryFMRP constructs and phoactivatable GFP (PAGFP)-FMRP constructs were generated at the Emory University Custom Cloning Core Facility. Neurons were transfected by Neuromag (OZ BIOSCIENCES), and Neuro2a cells with Lipofectamine 2000 (Invitrogen).

Neuron stimulation, immunofluorescence, and image quantitation. Hippocampal neurons (DIV 14-21) were stimulated with 3,5-dihydroxyphenylglycine (DHPG) $(50 \mu \mathrm{M})$ with or without pretreatment of okadaic acid (10 nM) or MG132 $(25 \mu \mathrm{M})$ for $20^{\prime}$. Neurons were fixed with $4 \%$ paraformaldehyde and processed for immunofluorescence to detect total [mouse-IC3 (Chemicon) or rabbit-A4055 (Sigma)] and phosphoFMRP (Antar et al., 2004; Narayanan et al., 2007). Images were acquired on a Nikon TE-ECLIPSE inverted microscope with a $60 \times$ differential interference contrast-oil objective. Multiple $z$-slices were acquired with a cooled CCD camera (Cascade, Photometrics), and intensity in distal regions $(>45 \mu \mathrm{m})$ of dendrites was quantitated.

Live cell imaging and analysis. Hippocampal neurons (DIV 9) were cotransfected with PAGFP-FMRP constructs and mCherry for morphologic identification of transfected cells. A distal region of the dendrite was selected for activation ( 405 lasers, six pulses, $40 \%$ laser power; A1R confocal microscope, Nikon). A brief pause (2 $\mathrm{min}$ ) was allowed for maturation of PAGFP. Subsequently, DHPG was added to the medium and images were immediately acquired (every $10 \mathrm{~s}$ for $8 \mathrm{~min}$ ). The PAGFP particles were automatically tracked by IMARISTrack software (Bitplane), and the intensities of the "spots" at each point were background subtracted. An unstimulated region proximal to the cell body was also measured to quantify the amount of fluorescence lost due to transport. Spots were classified as degrading if there was at least a $20 \%$ decrease in final intensities relative to initial fluorescence intensity. Relative fluorescence was calculated by normalizing the intensity to the initial intensity. The cumulative relative intensities were fitted to an exponential decay, and the degradation rates were extracted.

Immunoprecipitation and Western blotting. Immunoprecipitation (IP) was performed as before (Muddashetty et al., 2007) on high-density cortical neurons at DIV 14 incubated with MG132 (carbobenzoxy-L-leucyl-L-leucylL-leucinal) $(25 \mu \mathrm{M}, 12 \mathrm{~h})$ and treated with DHPG (1 min) before wash and lysis with IP buffer, with or without pretreatment with okadaic acid (10 nM). For ubiquitination assays, Neuro2a cells were cotransfected with GFPFLAG or mCherry $3 \times$ FLAG-fused FMRP constructs and HA-tagged ubiquitin $(24 \mathrm{~h})$. After preincubation with MG132 (4 h), cells were lysed and used for immunoprecipitation followed by Western blotting. Band intensities were quantified using ImageJ.

Luciferase assays. Relative luciferase activity of a reporter fused with the 3' UTR of PSD-95 was measured by dual luciferase assay, as described previously (Muddashetty et al., 2011). High-density cortical neurons were stimulated with DHPG for indicated times with or without okadaic acid and MG132 pretreatment.

$\leftarrow$

(Figure legend continued.) with DHPG and imaged every $10 \mathrm{~s}$ for $8 \mathrm{~min} . \boldsymbol{K}$, Sequential timelapse images of the activated region indicated in $J$ ) at indicated times of DHPG activation. The FMRP granules were tracked automatically as spots with variable areas (ImarisTrack). Bottom, Sequential images of a heat map of the spot intensities of selected granules. $L$, Sample traces of the relative intensities of selected PAGFP particles in $\boldsymbol{K}$ as a function of time after DHPG treatment. $\boldsymbol{M}$, Temporal variation of cumulative relative fluorescence intensities of multiple PAGFPFMRP granules $(n=60-80)$ under basal condition, DHPG treatment, or a pretreatment with okadaic acid or MG132 for $30 \mathrm{~min}$ were fitted to exponential decay as a function of time after DHPG, and the rate constants were extracted and are depicted in Table 1.
Table 1. Kinetics of degradation of FMRP fused to PAGFP under various conditions

\begin{tabular}{llllc}
\hline Protein & Condition & $t_{1 / 2}$ & $K_{\text {obs }}$ & $\begin{array}{l}\text { Particles } \\
\text { degraded (\%) }\end{array}$ \\
\hline PAGFP-FMRP & Basal & $>5 \mathrm{~h}$ & $3.64 \times 10^{-6} \mathrm{~s}^{-1}$ & 21.3 \\
& DHPG & $263 \mathrm{~s}$ & $2.63 \times 10^{-3} \mathrm{~s}^{-1}$ & 76.27 \\
& OA + DHPG & NA & $<10^{-8}$ & $<10$ \\
& MG132 & NA & $<10^{-8}$ & $<10$
\end{tabular}

Average ( $n \geq 60$ ) relative fluorescence intensities at each time point fitted as a function of time to an exponential decay. NA, Values not available; $0 \mathrm{~A}$, okadaic acid; $K_{\text {obs }}$ is first order rate constant; $t_{1 / 2}$, half-life.

\section{Results \\ FMRP degradation in dendrites by UPS is rapid and requires PP2A activity \\ While UPS activity has been implicated in mGluR-induced hip-} pocampal LTD (Hou et al., 2006) and epileptogenesis (Zhao et al., 2011), and FMRP is degraded after mGluR stimulation, it is not known whether FMRP degradation is a necessary step in this cascade to stimulate synaptic protein synthesis. We investigated whether mGluR-induced signaling of translation involves FMRP ubiquitination and degradation. Cultured hippocampal neurons were treated with DHPG, a gp 1 mGluR agonist, for $30 \mathrm{~s}$, and FMRP levels were quantified in distal dendrites by immunofluorescence with IC3 antibody. mGluR activation decreased the FMRP signal in dendrites $(33.1 \pm 3.629 \%)$ (Fig. $1 A-C)$. Pretreatment of neurons with a PP2A inhibitor, okadaic acid (Fig. 1C) or UPS inhibitors, MG132 (Fig. 1B) or bortezomib (Fig. 1E), occluded mGluR-induced loss of FMRP, suggesting that FMRP is degraded by the UPS system and requires FMRP dephosphorylation by PP2A (Fig. $1 B, C, E$ ). The rapid mGluR-induced loss of dendritic FMRP was also detected with a C-terminal anti-FMRP antibody (data not shown). Quantitative immunofluorescence with a phosphospecific antibody showed that PP2A-mediated dephosphorylation of FMRP was not dependent on UPS since DHPG-induced loss of the phospho-FMRP signal was not affected by pretreatment of neurons with MG132 (Fig. 1D). These results indicated that dephosphorylation and degradation of FMRP in dendrites are distinct events that can be uncoupled, and further suggest that PP2A activity is required for degradation.

mGluR activation was previously shown to result in a loss of FMRP signal from postsynaptic compartments of cultured neurons (Antar et al., 2004). Here, we used isolated synaptoneurosomes (SNSs) to examine the effect of mGluR activity on synaptic FMRP levels in the presence of anisomycin to inhibit protein synthesis. Western blot analysis of FMRP from SNSs indicated that, by 2 and 5 min after DHPG treatment, FMRP levels were significantly decreased ( $28.4 \pm 4.7 \%$ at $2 \mathrm{~min}, 45.14 \pm 15 \%$ at 5 $\mathrm{min}$ ) in the presence anisomycin (Fig. $1 F, G$ ). However, DHPGinduced loss of FMRP was absent in SNSs treated with MG132, suggesting a role for UPS (Fig. $1 F, G$ ). DHPG-induced loss of FMRP was dependent on signaling through mGluR5, since pretreatment of synaptoneurosomes with MPEP abolished FMRP loss (Fig. $1 H, I$ ).

To study DHPG-induced loss of FMRP while excluding potential effects arising from FMRP transport and synthesis, or antibody accessibility, we used photoactivatable FMRP in live neurons. PAGFP-FMRP was expressed in hippocampal neurons, and a small region of distal dendrites was activated using a 405 $\mathrm{nm}$ laser to reveal GFP-FMRP puncta in dendrites. The photoactivated GFP signal was monitored for $500 \mathrm{~s}$ following DHPG stimulation or vehicle. Loss of PAGFP-FMRP signal was detectable as soon as $2 \mathrm{~min}$ following photoactivation. A total of $21.3 \%$ of GFP-FMRP particles showed a decrease in signal intensity dur- 


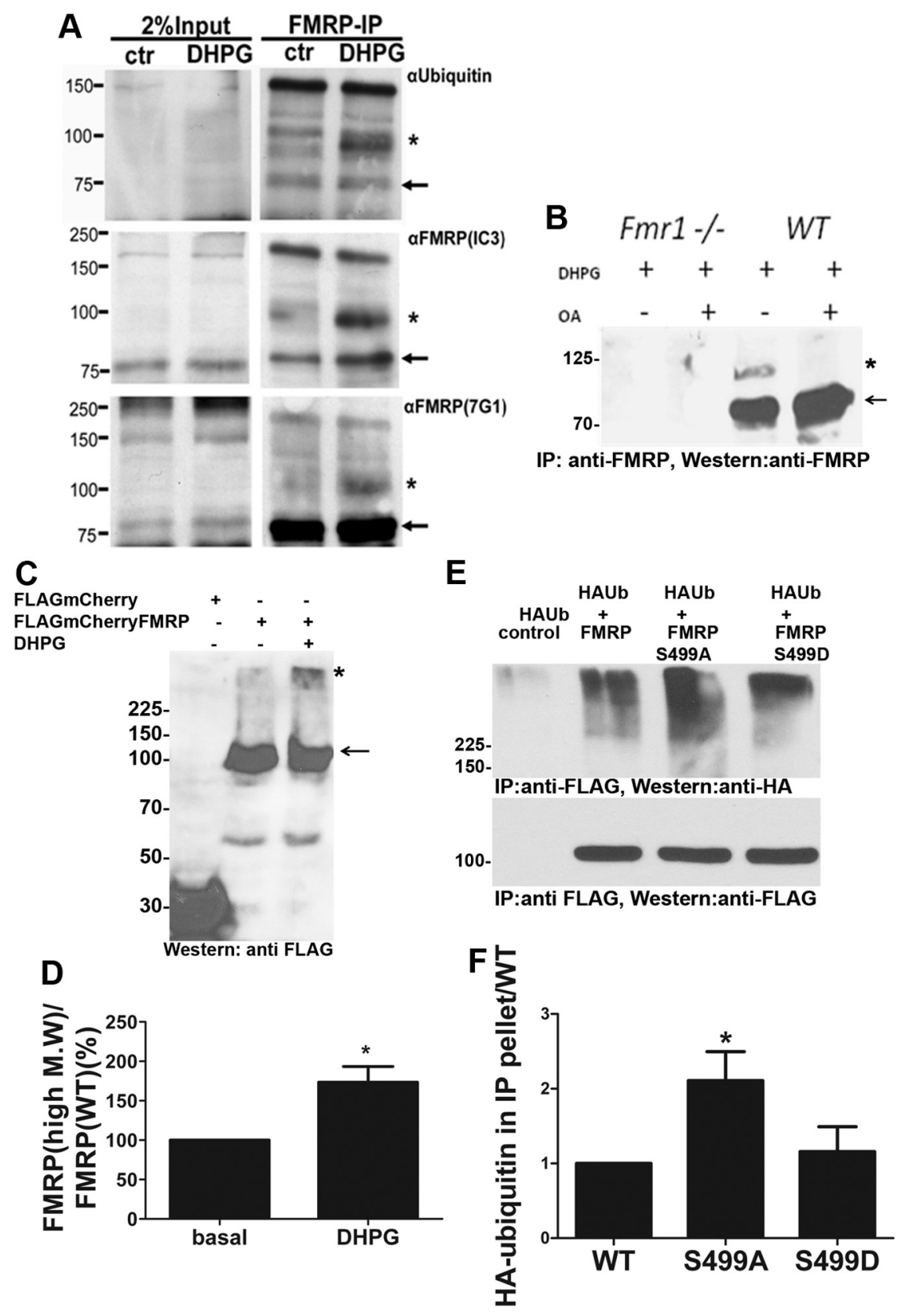

Figure 2. Rapid mGluR-induced polyubiquitination of FMRP requires dephosphorylation by PP2A at serine 499. A, Western blots performed with antibodies to different epitopes of FMRP in FMRP immunoprecipitates from cortical neurons pretreated with MG132 followed by DHPG for 1 min. *High-molecular-weight FMRP species; arrow, the unubiquitinated protein. $\boldsymbol{B}$, Western blots of FMRP immunoprecipitates from WT or Fmr 1 KO neurons pretreated with MG132 and with or without pretreatment with okadaic acid. *High-molecular-weight FMRP species; arrow, the unubiquitnated band. C, Western blots of lysates from Neuro2a cells expressing FLAGmCherry-FMRP or FLAGmCherry in the presence of MG132 with or without treatment with DHPG. *Highmolecular-weight FMRP species; arrow, the unubiquitinated protein. $\boldsymbol{D}$, Histogram of mean \pm SEM of ubiquitinated FMRP band intensities from $\boldsymbol{C}$ normalized to nonubiquitinated FMRP ( $n=4, p=0.01, t$ test). $\boldsymbol{E}, \boldsymbol{F}$, Mutation constructs of the primary phosphorylation site S499 (S499D, phosphomimic; S499A, dephosphomimic) and HA ubiquitin were cotransfected in Neuro2a cells. $\boldsymbol{E}$, Immunoprecipitated FMRP extracts screened by western blot with anti-HA antibody. $\boldsymbol{F}$, Histogram of mean \pm SEM of HA- ubiquitin smear intensities in the IP normalized first to FMRP in IP and then to HA ubiquitin in the lysate $(n=4, p=0.0332$, one-way ANOVA with Bonferroni's test).

ing time lapse in vehicle-treated neurons (Fig. $1 J-M$; Table 1), whereas addition of DHPG increased the percentage of degrading particles to $76.27 \%$. The time course of changes in relative intensities upon DHPG treatment could be fitted to a one-phase exponential decay with a half-life of $4.3 \mathrm{~min}$ (Fig. 1M; Table 1). Analysis of fluorescence intensities at a proximal $(0-5 \mu \mathrm{m}$ from cell body) nonactivated ROI showed no difference between the fluorescence intensities of basal and DHPG-treated neurons (data not shown), suggesting that DHPGinduced loss in the locally photoactivated FMRP signal in distal dendrites was not due to transport of photoactivated FMRP within dendrites during this very brief time period. Pretreatment of neurons with either okadaic acid or MG132 occluded the loss in the dendritic FMRP signal following DHPG treatment (Fig. $1 M$ ). This finding demonstrates that UPS-mediated dendritic FMRP degradation is increased by DHPG and requires PP2A activity.

Ubiquitination of FMRP is enhanced by mGluR activity and dephosphorylation We then investigated whether the loss of FMRP is due to FMRP ubiquitination. High-density cortical neurons pretreated with MG132 were used to immunoprecipitate endogenous FMRP. Stimulating neurons with DHPG (1 min) before lysis and immunoprecipitation led to an increase in the accumulation of a highmolecular-weight species of FMRP, which was also reactive to an anti-ubiquitin antibody (Fig. $2 A$ ). The $\sim 100 \mathrm{kDa}$ endogenous ubiquitinated FMRP species that appears upon DHPG treatment is in agreement with earlier reports using hippocampal slices (Hou et al., 2006). However, we could detect ubiquitination after very brief DHPG stimulation (1 $\mathrm{min}$ as opposed to $10 \mathrm{~min}$ in the previous study). This supports a model of immediate early UPS-mediated degradation of FMRP. These ubiquitinated FMRP bands could be detected by multiple FMRP antibodies. To further confirm this result, FLAGtagged FMRP was expressed in Neuro2a cells, which have previously been shown to be responsive to mGluR activation (Liu et al., 2002). Preincubation of transfected cells with MG132 led to an accumulation of high-molecular-weight species labeled by anti-FLAG antibody in cells expressing FLAGmCherry-FMRP but not FLAGmCherry alone (Fig. 2C). DHPG treatment further increased the proportion of FMRP in the high-molecular-weight smear $(73 \pm 19.8 \%)$ (Fig. $2 C, D$ ).

To assess the role of PP2A activity in polyubiquitination, cortical neurons from WT and Fmr1 KO neurons were pretreated with MG132, and with or without okadaic acid, before stimulating with DHPG. The endogenous high-molecular-weight FMRP species, which appeared only in the WT but not in the Fmr1 KO neurons, was significantly reduced in the presence of okadaic acid (Fig. $2 B$ ). We then analyzed whether the regulation of FMRP ubiquitination by PP2A was due to a change in FMRP phosphorylation by performing 

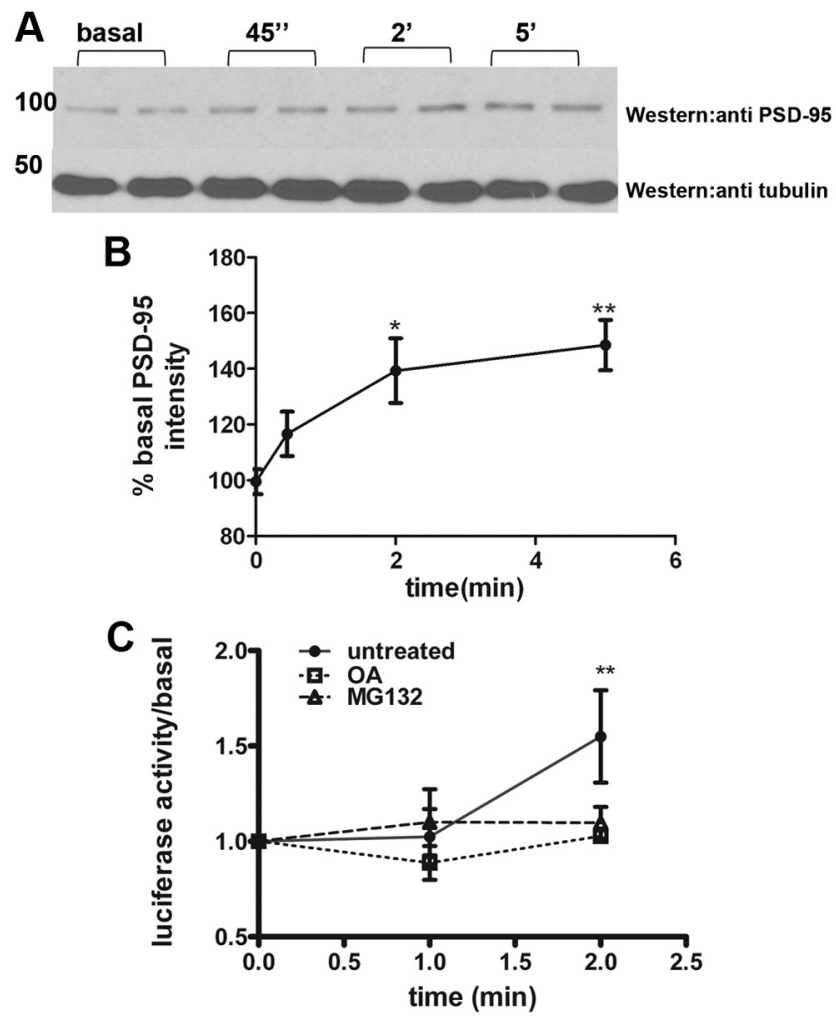

Figure 3. DHPG induction of PSD-95 expression is rapid and requires UPS and PP2A activity. A, Western blot of endogenous PSD-95 (top lane) in synaptoneurosomes treated with $50 \mu \mathrm{M}$ DHPG for indicated times. Tubulin is detected as loading control for normalization (bottom lanes). $\boldsymbol{B}$, Mean \pm SEM of normalized PSD-95 levels in SNSs as seen in $\boldsymbol{A}$ ) at indicated times after DHPG stimulation ( $n=6, p=0.0029$, one-way ANOVA with Bonferroni's test). C, Relative luciferase activity from cortical neurons transfected with f-luciferase-PSD-95 3' UTR construct. Neurons were stimulated for indicated times with $50 \mu \mathrm{M}$ DHPG with or without pretreatment with okadaic acid and MG132 for 30 min and relative luciferase activity was measured. The graph represents mean \pm SEM as a function of time after DHPG stimulation $(n=5, p=$ 0.0044, one-way ANOVA with Bonferroni's test).

ubiquitination assays with FMRP phosphomutants expressed in Neuro2a cells. FLAG-tagged phosphomutants of FMRP, which mimic the phosphorylated form (S499D) or dephosphorylated form (S499A) (Ceman et al., 2003), were coexpressed with HAubiquitin and immunoprecipitated with an anti-FLAG antibody. Western blot analysis with an antibody to HA revealed the accumulation of high-molecular-weight HA smear due to polyubiquitination in the anti-FLAG-FMRP immunoprecipitate (Fig. $2 E)$. These high-molecular-weight bands were also detected with an antibody to FLAG (data not shown). Dephospho-mimic S499A showed a significant increase in accumulation of polyHAubiquitin $(2.1 \pm 0.3$-fold $)$ compared with the WT or S499D (Fig. $2 E, F)$. This result suggests that the activity-induced UPSmediated degradation of FMRP is increased by dephosphorylation.

\section{Rapid mGluR-induced translation of PSD-95 mRNA requires activity of PP2A and UPS}

PSD-95 mRNA is a well studied FMRP target whose expression and translation have been shown to increase rapidly in response to mGluR activity (Todd et al., 2003; Muddashetty et al., 2007). We measured the endogenous levels of PSD-95 in SNSs by Western blot (Fig. 3A) and further showed that mGluR stimulation results in an increase in endogenous PSD-95 protein expression in isolated SNSs, occurring within $2 \mathrm{~min}(39.3 \pm 11.6 \%$ at $2 \mathrm{~min}$, $48.5 \pm 9.06 \%$ at $5 \mathrm{~min}$ ) (Fig. $3 B$ ), which is the same timescale as observed for FMRP degradation (Fig. 1E,F). Translation of a firefly luciferase reporter fused to the $3^{\prime}$ UTR of PSD-95 was shown to be dependent on the presence of FMRP (Muddashetty et al., 2011) and FMRP phosphorylation status, without affecting the steady-state levels of PSD-95 mRNA. The effect of inhibiting UPS and PP2A on this PSD-95 3' UTR reporter was examined. By 2 min of stimulation with DHPG, luciferase activity was increased 1.5-fold (Fig. 3C). Removal of the 3' UTR rendered the reporter unresponsive to DHPG, without alteration in steadystate mRNA levels (data not shown). Pretreament of neurons with okadaic acid or MG132 occluded the increased translation of PSD-95 mRNA in response to mGluR stimulation (Fig. 3C). Collectively, these data strongly suggest that translation of PSD-95 is tightly regulated by mGluR-mediated dephosphorylation and degradation of FMRP.

\section{Discussion}

Translational activation of FMRP target mRNAs has been found to be very rapid and to depend on FMRP dephosphorylation by PP2A (Narayanan et al., 2007). Here we show that dephosphorylation of FMRP by PP2A was required for FMRP degradation, which suggests coordinated molecular events that regulate the early steps of mGluR-induced translational induction. While other studies implicate FMRP degradation in response to mGluR activation (Hou et al., 2006; Zhao et al., 2011), it was not clear whether these molecular events, dephosphorylation and degradation, are coordinated and what concerted role they may play to rapidly activate translation. By detecting the accumulation of ubiquitinated FMRP and observing FMRP degradation in dendrites of live neurons in the same timescale as rapid PP2Ainduced dephosphorylation and PSD-95 translation induction, we provide evidence for rapid FMRP ubiquitination and degradation as a component of the early signaling events leading to rapid protein synthesis.

Our recent work also shows that FMRP dephosphorylation leads to disinhibition of translation by microRNA-induced silencing complex (miRISC) dissociation from mRNA (Muddashetty et al., 2011). A recent study has reported that FMRP inhibits translation by stalling ribosomes (Darnell et al., 2011). It is possible that regulation of translation by FMRP may involve multiple sequential and coordinated steps, which may be elicited first by dephosphorylation-mediated ubiquitination and miRISC release from mRNA followed by degradation of FMRP. We speculate that ubiquitination triggers release of RISC, removing translational inhibition within the microRNAribonucleoprotein complex, yet FMRP degradation is needed to remove ribosome stalling. Further work is needed to study the coordination and inter-relationships of these molecular events.

Our findings reveal a noncanonical mode of dephosphorylation-induced degradation of FMRP. The conventional degradation of phosphodegrons by SCF-Ring finger ubiquitin ligases proposes that phosphorylation in a PEST (peptides rich in Proline, Glutamate, Serine, and Threonine) instability sequence leads to degradation via ubiquitination of adjacent lysines (Rogers et al., 1986). Although a putative PEST site, a motif known to be involved in phosphorylation-based degradation exists in FMRP, overlapping with a phosphorylation site (serine 499) (Ceman et al., 2003), the half-life of FMRP expressed in fibroblasts did not seem to be changed by phosphorylation. It remains to be understood how dephosphorylation facilitates polyubiquitination as shown in our study. These questions could be answered from structural and proteomic studies. The mechanism may involve other UPS interacting proteins whose interaction may 
be increased by dephosphorylation. There are other studies that suggest phosphorylation prevents degradation. An N-terminal Proline in the c-mos degron promotes degradation by reducing phosphorylation at a downstream serine, which is a motif for an as yet unknown ligase (Sheng et al., 2002; Hunter, 2007). Dephosphorylation may cause a conformational change exposing the ligase recognition motif in FMRP or may cause changes in phosphorylation status of other residues. Moreover, PEST sites are highly variable in conformation and are involved in multiple cellular functions (Sandhu and Dash, 2006).

Protein homeostasis at the synapse and control of long-term plasticity involves the coordinate synthesis and degradation of synaptic proteins (Cajigas et al., 2010). mGluR-dependent synaptic plasticity appears to involve synthesis of proteins like Arc/ Arg3.1, MAP1B, and PSD-95 mRNAs, which are translationally regulated by FMRP. Herein we report a novel mechanism for UPS in dendrites linking the dephosphorylation-dependent degradation of FMRP to translational disinhibition of an FMRP target mRNA, PSD-95. These findings suggest a possible general mechanism to regulate protein synthesis-dependent synaptic plasticity by the degradation of a translational repressor.

\section{References}

Antar LN, Afroz R, Dictenberg JB, Carroll RC, Bassell GJ (2004) Metabotropic glutamate receptor activation regulates fragile $\mathrm{X}$ mental retardation protein and FMR1 mRNA localization differentially in dendrites and at synapses. J Neurosci 24:2648-2655.

Banerjee S, Neveu P, Kosik KS (2009) A coordinated local translational control point at the synapse involving relief from silencing and MOV10 degradation. Neuron 64:871-884.

Bingol B, Schuman EM (2004) A proteasome-sensitive connection between PSD-95 and GluR1 endocytosis. Neuropharmacology 47:755-763.

Cajigas IJ, Will T, Schuman EM (2010) Protein homeostasis and synaptic plasticity. EMBO J 29:2746-2752.

Ceman S, O’Donnell WT, Reed M, Patton S, Pohl J, Warren ST (2003) Phosphorylation influences the translation state of FMRP-associated polyribosomes. Hum Mol Genet 12:3295-3305.

Colledge M, Snyder EM, Crozier RA, Soderling JA, Jin Y, Langeberg LK, Lu H, Bear MF, Scott JD (2003) Ubiquitination regulates PSD-95 degradation and AMPA receptor surface expression. Neuron 40:595-607.

Darnell JC, Van Driesche SJ, Zhang C, Hung KY, Mele A, Fraser CE, Stone EF, Chen C, Fak JJ, Chi SW, Licatalosi DD, Richter JD, Darnell RB (2011) FMRP stalls ribosomal translocation on mRNAs linked to synaptic function and autism. Cell 146:247-261.

Ehlers MD (2003) Eppendorf 2003 prize-winning essay. Ubiquitin and the deconstruction of synapses. Science 302:800-801.

Fonseca R, Vabulas RM, Hartl FU, Bonhoeffer T, Nägerl UV (2006) A balance of protein synthesis and proteasome-dependent degradation determines the maintenance of LTP. Neuron 52:239-245.

Greer PL, Hanayama R, Bloodgood BL, Mardinly AR, Lipton DM, Flavell SW, Kim TK, Griffith EC, Waldon Z, Maehr R, Ploegh HL, Chowdhury S, Worley PF, Steen J, Greenberg ME (2010) The Angelman syndrome protein Ube3A regulates synapse development by ubiquitinating arc. Cell 140:704-716.

Hegde AN, Goldberg AL, Schwartz JH (1993) Regulatory subunits of cAMP-dependent protein kinases are degraded after conjugation to ubiquitin: a molecular mechanism underlying long-term synaptic plasticity. Proc Natl Acad Sci U S A 90:7436-7440.
Hershko A, Ciechanover A (1998) The ubiquitin system. Annu Rev Biochem 67:425-479.

Hou L, Antion MD, Hu D, Spencer CM, Paylor R, Klann E (2006) Dynamic translational and proteasomal regulation of fragile $\mathrm{X}$ mental retardation protein controls mGluR-dependent long-term depression. Neuron $51: 441-454$.

Hunter T (2007) The age of crosstalk: phosphorylation, ubiquitination, and beyond. Mol Cell 28:730-738.

Karpova A, Mikhaylova M, Thomas U, Knöpfel T, Behnisch T (2006) Involvement of protein synthesis and degradation in long-term potentiation of Schaffer collateral CA1 synapses. J Neurosci 26:4949-4955.

Liu F, Virshup DM, Nairn AC, Greengard P (2002) Mechanism of regulation of casein kinase I activity by group I metabotropic glutamate receptors. J Biol Chem 277:45393-45399.

Muddashetty RS, Kelić S, Gross C, Xu M, Bassell GJ (2007) Dysregulated metabotropic glutamate receptor-dependent translation of AMPA receptor and postsynaptic density-95 mRNAs at synapses in a mouse model of fragile X syndrome. J Neurosci 27:5338-5348.

Muddashetty RS, Nalavadi VC, Gross C, Yao X, Xing L, Laur O, Warren ST, Bassell GJ (2011) Reversible inhibition of PSD-95 mRNA translation by miR-125a, FMRP phosphorylation, and mGluR signaling. Mol Cell 42:673-688.

Narayanan U, Nalavadi V, Nakamoto M, Pallas DC, Ceman S, Bassell GJ, Warren ST (2007) FMRP phosphorylation reveals an immediate-early signaling pathway triggered by group I mGluR and mediated by PP2A. J Neurosci 27:14349-14357.

Pierce NW, Kleiger G, Shan SO, Deshaies RJ (2009) Detection of sequential polyubiquitylation on a millisecond timescale. Nature 462:615-619.

Rechsteiner M (1987) Ubiquitin-mediated pathways for intracellular proteolysis. Annu Rev Cell Biol 3:1-30.

Rinetti GV, Schweizer FE (2010) Ubiquitination acutely regulates presynaptic neurotransmitter release in mammalian neurons. J Neurosci 30:31573166.

Rogers S, Wells R, Rechsteiner M (1986) Amino acid sequences common to rapidly degraded proteins: the PEST hypothesis. Science 234:364-368.

Ross CA, Poirier MA (2004) Protein aggregation and neurodegenerative disease. Nat Med 10 [Suppl]:S10-S17.

Sandhu KS, Dash D (2006) Conformational flexibility may explain multiple cellular roles of PEST motifs. Proteins 63:727-732.

Sheng J, Kumagai A, Dunphy WG, Varshavsky A (2002) Dissection of c-MOS degron. EMBO J 21:6061-6071.

Steward O, Schuman EM (2003) Compartmentalized synthesis and degradation of proteins in neurons. Neuron 40:347-359.

Todd PK, Mack KJ, Malter JS (2003) The fragile X mental retardation protein is required for type-I metabotropic glutamate receptor-dependent translation of PSD-95. Proc Natl Acad Sci U S A 100:14374-14378.

Wang DO, Martin KC, Zukin RS (2010) Spatially restricting gene expression by local translation at synapses. Trends Neurosci 33:173-182.

Waung MW, Huber KM (2009) Protein translation in synaptic plasticity: mGluR-LTD, Fragile X. Curr Opin Neurobiol 19:319-326.

Weiler IJ, Irwin SA, Klintsova AY, Spencer CM, Brazelton AD, Miyashiro K, Comery TA, Patel B, Eberwine J, Greenough WT (1997) Fragile X mental retardation protein is translated near synapses in response to neurotransmitter activation. Proc Natl Acad Sci U S A 94:5395-5400.

Zalfa F, Giorgi M, Primerano B, Moro A, Di Penta A, Reis S, Oostra B, Bagni C (2003) The fragile X syndrome protein FMRP associates with BC1 RNA and regulates the translation of specific mRNAs at synapses. Cell 112:317-327.

Zhao W, Chuang SC, Bianchi R, Wong RK (2011) Dual regulation of fragile $\mathrm{X}$ mental retardation protein by group I metabotropic glutamate receptors controls translation-dependent epileptogenesis in the hippocampus. J Neurosci 31:725-734. 